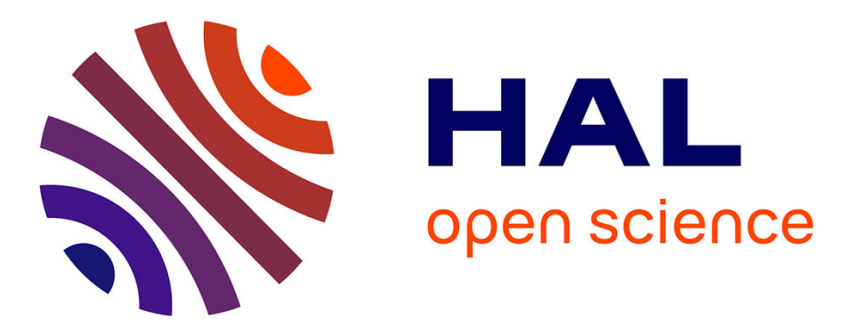

\title{
Characterization of the dynamics of hepatitis B virus resistance to adefovir by ultra-deep pyrosequencing.
}

Christophe Rodriguez, Stéphane Chevaliez, Paul Bensadoun, Jean-Michel Pawlotsky

\section{To cite this version:}

Christophe Rodriguez, Stéphane Chevaliez, Paul Bensadoun, Jean-Michel Pawlotsky. Characterization of the dynamics of hepatitis B virus resistance to adefovir by ultra-deep pyrosequencing.. Hepatology, 2013, 58 (3), pp.890-901. 10.1002/hep.26383 . inserm-00936562

\section{HAL Id: inserm-00936562 https://www.hal.inserm.fr/inserm-00936562}

Submitted on 30 Jun 2014

HAL is a multi-disciplinary open access archive for the deposit and dissemination of scientific research documents, whether they are published or not. The documents may come from teaching and research institutions in France or abroad, or from public or private research centers.
L'archive ouverte pluridisciplinaire HAL, est destinée au dépôt et à la diffusion de documents scientifiques de niveau recherche, publiés ou non, émanant des établissements d'enseignement et de recherche français ou étrangers, des laboratoires publics ou privés. 


\section{HEPATOLOGY}

\section{Characterization of the dynamics of hepatitis B virus resistance to adefovir by ultra-deep pyrosequencing}

\begin{tabular}{|r|l|}
\hline Journal: & Hepatology \\
\hline Manuscript ID: & HEP-12-2283.R1 \\
\hline Wiley - Manuscript type: & Original \\
\hline Date Submitted by the Author: & n/a \\
\hline Complete List of Authors: & $\begin{array}{l}\text { Rodriguez, Christophe } \\
\text { Chevaliez, Stephane; Hopital Henri Mondor, Service de Baceriologie- } \\
\text { Virologie } \\
\text { Bensadoun, Paul; inserm, U955 } \\
\text { Pawlotsky, Jean-Michel; Hopital Henri Mondor, Department of Virology }\end{array}$ \\
\hline Keywords: & hepatitis B virus, adefovir, resistance, ultra-deep pyrosequencing \\
\hline & \\
\hline
\end{tabular}

SCHOLARONE ${ }^{\text {Ix }}$

Manuscripts 


\title{
Characterization of the Dynamics of Hepatitis B Virus
}

\section{Resistance to Adefovir by Ultra-Deep Pyrosequencing}

\author{
Christophe Rodriguez, ${ }^{1,2}$ Stéphane Chevaliez,, ${ }^{1,2}$ Paul Bensadoun, ${ }^{2}$ \\ and Jean-Michel Pawlotsky ${ }^{1,2}$ \\ ${ }^{1}$ National Reference Center for Viral Hepatitis B, C and Delta, Department of Virology, \\ Hôpital Henri Mondor, Université Paris-Est, Créteil, France; \\ 2INSERM U955, Créteil, France
}

Key-words : hepatitis B virus, adefovir, resistance, ultra-deep pyrosequencing 


\title{
FOOTNOTE PAGE
}

Corresponding author: Professor Jean-Michel Pawlotsky, MD, PhD, Department of Virology, Hôpital Henri Mondor, 51 avenue du Maréchal de Lattre de Tassigny, 94010 Créteil, France. $\underline{\text { Tel: }}$ +33-1-4981-2827; $\underline{\text { Fax: }}$ +33-1-4981-4831; E-mail: jeanmichel.pawlotsky@hmn.aphp.fr

\begin{abstract}
Abbreviations: HBV: hepatitis B virus; PCR: polymerase chain reaction; UDPS: ultradeep pyrosequencing; HBeAg: hepatitis B e antigen; MID: multiplex identifier; HBsAg: hepatitis B surface antigen.
\end{abstract}

Financial support: This work was funded by the French National Agency for Research on AIDS and Viral Hepatitis (ANRS). We are particularly grateful to Prof. Jean-François Delfraissy, the Director of ANRS, for his continuous support to our UDPS projects. Christophe Rodriguez is the recipient of a doctoral fellowship from ANRS. The hosting laboratory is funded by the Fondation pour la Recherche Médicale (FRM) as an "FRM Team".

Conflicts of interest: C.R., S.C. and J.M.P. have received research grants from Gilead Sciences ; S.C. and J.M.P. have served as advisors for Gilead Sciences. 
ABSTRACT

Hepatitis B virus (HBV) resistance to nucleoside/nucleotide analogues is frequent. Ultra-deep pyrosequencing (UDPS) is a powerful new tool that can detect minor viral variants and characterize complex quasispecies mixtures. We used UDPS to analyze the dynamics of adefovir-resistant HBV variants in patients with chronic HBV infection in whom adefovir resistance occurred during treatment. Amino acid substitutions known to confer resistance to adefovir were detected at baseline in most of the patients. The dynamics of adefovir-resistant variants were complex and differed among the patients, as a result of evolving differences in variant fitness. UDPS analysis revealed successive waves of selection of HBV populations with single and multiple amino acid substitutions. Adefovir-resistant variants were partially inhibited by lamivudine but remained fit in its presence. In conclusion, we show that substitutions conferring HBV resistance to nucleoside/nucleotide analogues exist before treatment and that the dynamics of adefovir-resistant populations are much more complex and heterogeneous than previously thought and involve so far unknown amino acid substitutions. The UDPS-based approach described here is likely to have important implications for the assessment of antiviral drug resistance in research and clinical practice. 


\section{INTRODUCTION}

Approximately 240 million individuals worldwide are chronically infected with hepatitis B virus (HBV) (1). Chronic HBV infection is the leading cause of chronic liver disease and accounts for nearly a million deaths every year (2-4). Chronic hepatitis B can be treated with either pegylated interferon- $\alpha$ or nucleoside/nucleotide analogues. The latter drugs act by directly inhibiting the enzymatic function of HBV reverse transcriptase, the enzyme responsible for viral replication. Five such drugs have been approved for HBV therapy, namely three nucleoside analogues (lamivudine, telbivudine and entecavir) and two nucleotide analogues administered as prodrugs (adefovir dipivoxil and tenofovir disoproxil fumarate). The vast majority of HBV-infected patients have an indication for therapy with nucleoside/nucleotide analogues.

The main issues with nucleoside/nucleotide analogues are the need for long-term (probably life-long) administration and the possible emergence of viral resistance (5). Resistance is characterized by outgrowth of viral populations bearing amino acid substitutions that confer reduced sensitivity to the drug. This is due to the quasispecies distribution of HBV in infected individuals, i.e. the coexistence of a mixture of genetically distinct but closely related viral populations in an unstable equilibrium that depends strongly on their relative fitness (i.e. their ability to propagate efficiently) in a specific replicative environment (5-7). Resistant variants that emerge during treatment are thought to pre-exist as minor populations prior to treatment, but this remains to be demonstrated in the case of HBV. The fitness cost of drug resistance can gradually be offset by the accumulation of "compensatory" amino acid substitutions during replication (5-7). HBV resistance generally results in virological and biochemical breakthrough, followed by accelerated liver disease progression $(5,6)$. 
Few techniques are available to study HBV resistance in the clinical setting. Population sequencing (or direct sequencing) is the most widely used, but it can only detect the dominant viral population(s). Reverse hybridization with the line probe assay can only detect variants representing at least $5 \%$ of the viral quasispecies, and can only identify substitutions already known to confer $\operatorname{HBV}$ resistance to a given drug $(8,9)$. Sequencing of multiple clones generated after polymerase chain reaction (PCR) amplification is cumbersome and time-consuming (10-13). In addition, analysis of 20 clones per time point provides only a $95 \%$ probability that variants representing $10 \%$ or more of the viral quasispecies will be identified, whereas random minor variants with no clinical significance may also be highlighted with this method. Novel technical approaches are therefore needed to study antiviral drug resistance.

Next-generation sequencing techniques are capable of generating vast quantities of data without prior knowledge of a particular gene or sequence of interest. The 454 technology (454 Life Sciences, Roche Diagnostics Corp., Branford, CT), based on ultradeep pyrosequencing (UDPS), provides longer reads than most other techniques and is well suited to viral resistance studies (14-17).

Adefovir dipivoxil is still used as first-line monotherapy or as rescue therapy after lamivudine treatment failure in a very large number of HBV-infected patients in settings or areas of the world where more potent drugs, such as tenofovir or entecavir, are not approved or not affordable by the majority of the population. In this context, we used an original approach based on UDPS to characterize HBV genetic variability at baseline and the dynamics of adefovir-resistant HBV variants in patients receiving this therapy, alone or combined with lamivudine in case of adefovir treatment failure.

\section{MATERIALS AND METHODS}




\section{Patients}

The study patients were selected from a 240 -week clinical trial of adefovir monotherapy in nucleoside/nucleotide-naïve hepatitis B e antigen (HBeAg)-negative patients, with a double-blinded phase of 96 weeks followed by an open-label, long-term safety and efficacy study of 144 weeks (18). Serial HBV DNA level measurements were systematically performed during therapy, as well as population sequencing of the fulllength HBV reverse transcriptase gene at baseline and, in patients with detectable serum HBV DNA, at weeks 48, 96, 144, 192, and 240. Resistance-associated substitutions were selected in 29 patients during the 240 -week study. Their viral dynamics classified them into three groups: responders (HBV DNA level reduction of more than $3 \log _{10}$ international units [IU]/mL) who experienced secondary treatment failure (reincrease in the HBV DNA level of more than $1 \log _{10} \mathrm{IU} / \mathrm{mL}$ above the nadir); suboptimal responders (HBV DNA level reduction of less than $3 \log _{10} \mathrm{IU} / \mathrm{mL}$ ) who developed amino acid substitutions in a context of a slow, gradual re-increase in viral replication; and responders who developed the amino acid substitutions without any virological breakthroughs. We selected the first seven patients displaying these three dynamic patterns in order to study HBV population dynamics during adefovir exposure.

The 7 patients were 5 men and 2 women, 26 to 59 years old. Their characteristics, including serial reverse transcriptase sequence analysis by means of cloning-sequencing, have been described elsewhere (11). Correspondence with the numbering in the previous article is shown in Supplementary Table 1. None of them had previously received nucleoside/nucleotide analogue treatment, and all received 10 $\mathrm{mg} /$ day adefovir for the full study period. In four cases (patients 1, 2, 5 and 7), 
lamivudine was added during adefovir therapy because of virological failure. Serial serum samples taken at baseline and during adefovir therapy were analyzed by UDPS. The data were then analyzed and interpreted with a specific software package.

Two independent groups of patient were used as comparators for the frequency of amino acid substitutions associated with adefovir resistance at baseline. The first group included 5 treatment-naïve, HBeAg-positive patients ( 3 men, 2 women, 15 to 18 years old) treated with adefovir, $10 \mathrm{mg} /$ day (104 weeks in 3 cases, 196 weeks in 2 cases) who responded to therapy and achieved an HBe seroconversion with sustained undetectable HBV DNA after therapy. These patients were randomly selected from a larger group of patients included in a previously reported study reporting the safety, efficacy, and pharmacokinetics of adefovir dipivoxil in children and adolescents with chronic hepatitis B who responded to this therapy (19). The second group included 11 treatment-naïve patients with chronic hepatitis B (22 to 60 years old), consecutively seen for the first time in the Department of Hepatology of the University Hospital of Caen, France, during the 2008-2011 period. The baseline samples were analyzed by UDPS and the results were interpreted with the same software package.

\section{First HBV DNA PCR round}

HBV DNA was extracted from 200-500 $\mu \mathrm{L}$ of every available serum sample with the QIAamp DNA blood kit (Qiagen GmbH, Hilden, Germany), according to the manufacturer's instructions. A 630-bp fragment encompassing domains A to E of HBV reverse transcriptase was PCR-amplified with primers pol1 and pol2, as previously described $(11,20)$. 


\section{Controls}

The first-round PCR amplicon from patient 1's baseline sample was cloned into TOPO TA Cloning 2.1 vector (Invitrogen, Carlsbad, CA), transformed by means of One Shot ${ }^{\circledR}$ TOP10 chemically competent Escherichia Coli (Invitrogen) and cultured in brain heart infusion agar Petri dishes with $1 \mathrm{mg} / \mathrm{mL}$ ampicilline. Ten colonies were sequenced with M13 primers, according to the TOPO TA cloning protocol (Invitrogen). The sequences were aligned with ClustalX v2.0.9. One wild-type colony was selected and amplified into BHI medium containing $1 \mathrm{mg} / \mathrm{mL}$ ampicilline. It was then purified by means of PureLink ${ }^{\mathrm{TM}}$ HiPure Plasmid Filter Maxiprep Kit (Invitrogen). Plasmid DNA was quantified by means of Quant-iT ${ }^{\mathrm{TM}}$ dsDNA Assay Kit (Invitrogen) and diluted in order to achieve final concentrations of $10^{8}, 10^{5}$, and $5 \times 10^{3}$ copies/mL. The final DNA amounts were confirmed by means of a quantitative real-time PCR technique on ABI 7500 (Applied Biosystems, Carlsbad, CA). Each plasmid dilution was then amplified in triplicate in three independent PCR reactions using primers pol1 and pol2, as described above. The 9 controls at three dilutions were used to calculate the error rate of the $\underline{\text { technique at each amino acid position. }}$

\section{Ultra-deep pyrosequencing}

A second "nested" PCR amplification was performed with internal primers pol3 and pol4 (11) that were modified to introduce a GS FLX bead adaptor and a specific identity tag (multiplex identifier, MID). A combination of 8 different MIDs was used to identify each sample. Amplicons containing the bead adaptor and MID were then purified in Nucleofast ${ }^{\circledR} 96$ PCR plates (Clontech, Moutain View, CA), according to the 
manufacturer's instructions. The amplicons were then quantified with the Quant-iT ${ }^{\mathrm{TM}}$ PicoGreen ${ }^{\circledR}$ dsDNA kit (Invitrogen), fixed to beads, and amplified in a microemulsion with the GS FLX Titanium emPCR kit (454 Life Sciences). The amplified beads were purified and enriched according to the manufacturer's instructions, counted with a Beckman Coulter Z1 particle counter (Beckman Coulter, Brea, CA), and deposited in a GS FLX Titanium PicoTiterPlate (454 Life Sciences). The pyrosequencing reaction was performed with the GS FLX Titanium sequencing kit on an FLX Genome Sequencer (454 Life Sciences).

\section{Computer analysis of UDPS data}

Data generated with the UDPS method were analyzed with four in-house software programs included in the PyroPack $^{\odot}$ package, including PyroClass $^{\odot}$, PyroMute ${ }^{\odot}$, PyroDyn $^{\circledR}$, and PyroLink ${ }^{\odot}$, designed respectively to classify, filter, model and link viral sequences generated with these methods.

Sequence data analysis with PyroPack ${ }^{\odot}$ is based on the following procedure. Sequences generated by the GS-FLX sequencer are merged into a single file. Each sequence is identified by means of its sample tag and assigned to the file corresponding to the sample of origin by PyroClass ${ }^{\circledR}$. PyroMute ${ }^{\circledR}$ then uses a number of quality filters to eliminate unreliable sequences. Sequence portions with a low Phred quality score are removed (21). Too short sequences $(<50 \mathrm{bp})$, including those generated by the previous filtering step, are also eliminated. Sequences are then aligned by means of a modified version of the Smith-Waterman algorithm (22), in which the resolution of alignment matrices is accelerated and the identification of insertions/deletions (so-called indels) and the correction of length errors in homopolymeric sequences are improved. Quality 
filters subsequently remove sequences with an identity score $<80 \%$ relative to the consensus sequence of the patient's baseline sample. In the next step, an array of nucleotide substitutions and corresponding Phred quality scores is built and tested by means of a modified statistical test based on the binomial law (23), in order to eliminate sequences that are too rare and/or of poor quality, likely due to sequencing errors. Finally, the remaining nucleotide sequences, considered reliable, are converted into amino acid sequences and their respective frequencies are calculated. Each amino acid change is ascribed to its sequence of origin in order to subsequently analyze linkages between substitutions.

PyroDyn $^{\circledR}$ uses data generated by PyroMute ${ }^{\circledR}$ to detect and quantify increases or decreases in amino acid substitutions through mathematical modeling of their variations and correlation with an exponential growth model, which provided the best fit with the observed data. Briefly, in every patient, the frequency of each amino acid at each position is established at each time point. Assuming that HBV resistance is governed by exponential outgrowth of selected resistant variants, the best-fit curve is automatically drawn for each substitution in each patient. Combined cut-off values have been established $\left(r^{2}>0.8\right.$ and growth rate $>$ mean of the growth rates of all substitutions at all positions plus 2 standard deviations) to differentiate significant exponential changes from polymorphism fluctuations.

Finally, PyroLink ${ }^{\odot}$ has been designed to analyze genetic linkages between amino acid substitutions which have been selected by PyroDyn ${ }^{\circledR}$ and to characterize the dynamics of viral populations bearing one or several amino acid substitutions over time. Briefly, linkages are automatically sought for every substitution identified with PyroDyn ${ }^{\circledR}$ as exponentially growing or decreasing over time. Then, sequences that span all of the identified substitutions are extracted from PyroMute ${ }^{\odot}$ data and, when more 
than 100 such sequences are available, the proportion of sequences bearing no (wildtype), one, 2, 3, etc, substitutions is calculated and used for subsequent analyses.

\section{RESULTS}

\section{UDPS sequence data}

UDPS analysis of the 9 controls described in the Methods section and tested at different concentrations revealed that the mean maximum error rate generated by the technology was $0.120 \pm 0.005 \%$. However, this number varied according to the considered amino acid position. Therefore, only substitution frequencies higher than the mean maximal error rate for the corresponding amino acid position plus two standard deviations were taken into account in the analysis. The plasmids were tested in triplicate at different dilutions ranging from $5 \times 10^{3}$ to $10^{8}$ copies $/ \mathrm{mL}$. No impact of the DNA amount on UDPS results was observed (data not shown).

UDPS was then applied to serum samples taken at baseline and frequently during therapy, representing a total of 119 serial samples from 7 patients who developed resistance to adefovir monotherapy (15 to 24 samples per patient). Approximately 480,000 sequences (111 Mbp) were generated, with $4010 \pm 843$ sequences per sample and a mean length of $382 \pm 31 \mathrm{nt}$ after eliminating excessively short sequences $(\leq 50 \mathrm{nt})$. Overall, $10.2 \%$ of the generated sequences were eliminated by the software because of inadequate quality.

Presence of amino acid substitutions known to confer HBV resistance to nucleoside/nucleotide analogues in baseline samples 
Table 1 shows the prevalence of amino acid substitutions known to confer HBV resistance to nucleoside/nucleotide analogues detected by UDPS at baseline in the 7 patients. Five samples were found to harbor rtA181V/T substitutions, one harbored the rtN236T substitution, and two harbored rtM204V/I substitutions. No substitutions known to improve the fitness of rtM204V/I variants in the presence of lamivudine, telbivudine or entecavir (rtV173L and rtL180M) were found in the baseline samples. One sample harbored the rtT184S/A/I/L substitution and none the rtR202G substitution; both these substitutions are known to confer full resistance to entecavir when associated with rtM204V/I and rtL180M.

Table 1 also shows the prevalence of amino acid substitutions known to confer HBV resistance to nucleoside/nucleotide analogues detected by UDPS in the two comparator groups described in the Methods section. Among the 5 HBeAg-positive patients who seroconverted and maintained undetectable HBV DNA levels after adefovir therapy, two were found to harbor rtA181V/T substitutions at baseline, whereas none harbored the rtN236T substitution; two harbored rtM204V/I substitutions, including one in whom it represented nearly $10 \%$ of the viral quasispecies and was associated with an rtL180M substitution, suggesting prior exposure to lamivudine; rtT184S/A/I/L and rtR202G substitutions were found in one and one patient, respectively. Among the 11 unselected treatment-naive patients with chronic hepatitis B consecutively seen for the first time in the Department of Hepatology of the University Hospital of Caen, three were found to harbor rtA181V/T substitutions (representing more than $11 \%$ of the viral quasispecies in one case), and 6 harbored the rtN236T substitution (representing more than $3 \%$ of the viral quasispecies in one case); rtM204V/I substitutions were present in 
5 patients and rtT184S/A/I/L in 5 patients, whereas none harbored rtV173L, rtL180M or rtR202G substitutions at baseline.

\section{Characterization of the dynamics of HBV resistance to adefovir in patient 1}

Patient 1 responded suboptimally to adefovir, and the HBV DNA level started to increase gradually after a nadir at month 6, until the end of follow-up at month 24 . Figure $1 \mathrm{~A}$ shows the time course of the HBV DNA level, together with the dynamics of HBV viral populations during adefovir therapy in this patient, as assessed by UDPS. The results are presented as the absolute amount of each viral variant, in $\log _{10} \mathrm{IU} / \mathrm{mL}$, at each time point, taking into consideration the reverse transcriptase sequence only. The findings in Figure 1A can be summarized as follows. (i) Immediately after treatment initiation, we observed the persistence of minor variants with the single amino acid substitutions rtN138K, rtR139K and rtR212T that were present at baseline and remained quantitatively unchanged during adefovir administration, while the wild-type virus was profoundly inhibited. (ii) Immediately after the HBV DNA nadir was reached at month 2, the absolute amount of wild-type virus started to increase again, whereas the minor variants gradually lost their relative fitness and became nearly undetectable when outgrowth of adefovir-resistant variants started to be observed. (iii) The first wave of resistant variant outgrowth was detected at month 17 and peaked at months 21-22, when the wild-type virus became undetectable. This wave was composed of viral variants bearing single amino acid substitutions known to confer adefovir resistance, including a majority of rtN236T and a minority of rtA181T. (iv) A second wave of outgrowth of adefovir-resistant variants then gradually replaced the first wave, probably following fitness acquisition by resistant variants bearing single and double 
amino acid substitutions, including, by order of frequency, rtN236T+rtA181T, rtY245H, rtN236T+rtY245H, and rtN236T+rtD238N.

Figure 1B shows combined analysis of UDPS data on both the reverse transcriptase and HBsAg domains. As expected, the rtA181V substitution was systematically associated with an sL173F substitution in the hepatitis B surface antigen (HBsAg) sequence, due to the overlapping nature of the open reading frames coding for both viral proteins. The rtA181T substitution was associated with changes at position sW172; their distribution remained stable over time in this patient, with approximately $80 \%-90 \%$ of sW172* (stop codon) and 10\%-20\% of sW172L. In addition, HBsAg substitutions not encoded by the nucleotide changes responsible for substitutions in the reverse transcriptase region were linked to reverse transcriptase substitutions selected by adefovir (sS143T with rtA181T and sM197T with rtN236T). Variants bearing the $\underline{\text { s143T and sM197T substitutions were present at very low level at baseline. The double }}$ $\underline{\text { sM197T }+r t N 236 T \text { variant emerged and outgrew at the time of virological breakthrough }}$ (Figure 1B). In contrast, outgrowth of the triple sS143T+rtA181T(sW172L/*)+rtN236T variant followed outgrowth of the single sS143T variant by 4 months, both variants being present in equivalent amounts at the end of follow-up (data not shown). Both s143T and sM197T HBsAg substitutions appeared to contribute to the fitness gain of the second wave of adefovir-resistant variants, with preferential outgrowth of sM197T+rtN236T and sS143T+rtA181T(sW172L/*)+rtN236T variants over single or other multiple adefovir-resistant mutants at this late stage of follow-up (Figure 1B).

\section{Characterization of the dynamics of $\mathrm{HBV}$ resistance to adefovir in the other}

\section{6 patients}


The results of UDPS analyses in the other 6 patients are shown in Figure 2 and summarized below. Contrary to patient 1 , amino acid substitutions were not selected in the HBsAg region in the other patients, except those at positions sW172 and sL173 associated with $\mathrm{rtA} 181 \mathrm{~V} / \mathrm{T}$ when present.

Patient 2 was a suboptimal responder who experienced a slow, gradual reincrease in viral replication. In this patient (Figure 2A), wild-type HBV declined gradually during adefovir administration but re-increased when treatment was stopped after approximately a year. When adefovir was reintroduced a few weeks later, wildtype HBV declined again slowly and plateaued at approximately $10^{4} \mathrm{IU} / \mathrm{mL}$. The emergence of resistance was characterized by simultaneous selection of variants with the single rtN236T and rtA181V(sL173F) substitutions at week 27. Subsequently, the rtA181V(sL173F) variant became predominant and was responsible for the virological breakthrough. This variant was partially inhibited, but remained dominant, when lamivudine was added to adefovir after 43 months of therapy.

Patient 3 was a responder who experienced a virological breakthrough. In this patient (Figure 2B), resistance occurred at month 29 and was characterized by initial outgrowth of HBV variants with single or double amino acid substitutions at positions rtA181 and rtN236. In contrast to patient 2, a variant with the single rtN236T substitution took over and was responsible for the virological breakthrough. As in patient 2 , this variant was partially inhibited by lamivudine but remained predominant on combination therapy.

Patient 4 exhibited a mixed virological response pattern and a more complex resistance pattern (Figure 2C). This patient had a suboptimal response to adefovir. During the plateau phase, which lasted approximately 20 months, with mild fluctuations, wild-type HBV was gradually replaced by a mixture of variants with single (rtY124H or 
rtN236T), double (rtY124H+rtN236T) and triple (rtY124H+rtN236T+rtN238T) amino acid substitutions that replicated at low levels. Wild-type virus returned when adefovir treatment was interrupted. Adefovir was reintroduced about 2 months later, and resistance then developed, along with a typical virological breakthrough due to outgrowth of a viral population bearing the single rtN236T substitution. This variant was partially inhibited by lamivudine. Subsequently, on adefovir-lamivudine combination therapy, the same mixture of single, double and triple variants as observed earlier during therapy emerged and replicated at a low level.

Patient 5 (Figure 2D) exhibited a simple resistance pattern, characterized by a suboptimal response to adefovir and virological breakthrough at month 37 , due to outgrowth of a resistant variant bearing two amino acid substitutions $($ rtA181V(sL173F $)+r t N 236 T)$

Patient 6 (Figure 2E) responded to adefovir but low-level viremia persisted, with transient selection of a variant bearing an rtN238E substitution. Subsequently, a variant bearing the double rtN236T+rtN238E substitution took over and persisted at a low level (about $10^{2} \mathrm{IU} / \mathrm{mL}$ ).

Finally, in patient 7 who initially responded and subsequently broke through (Figure 2F), the virological breakthrough was related to the selection of a major viral population bearing the $\operatorname{rtA181V(sL173F)~substitution~and~a~minor~population~bearing~}$ the double rtA181V(sL173F)+rtF221Y substitution. Both variants were partially inhibited but remained dominant when lamivudine was added to adefovir.

\section{DISCUSSION}


Drug resistance is the principal cause of antiviral treatment failure, which may result in clinical disease progression. Next-generation sequencing technologies, such as UDPS, have the capacity to generate thousands of sequences from complex genomic mixtures, including sequences from dominant, intermediate and minor viral populations $(24,25)$. UDPS-based GS FLX technology provides sequence reads of sufficient length to span the region of interest when studying HBV resistance to nucleoside/nucleotide analogues. To interpret the data generated with this method, we developed an original package of four complementary software programs capable of analyzing large numbers of sequences (nearly 500,000 in this study) in the specific context of viral resistance, and used it in this study.

The HBV DNA level in the starting sample may theoretically have an impact on the sensitivity of detection of minor quasispecies variants by UDPS. This did not influence our description of baseline distributions of HBV variants because all patients had high viral levels in the absence of therapy. On adefovir or adefovir-lamivudine treatment, we were able to generate sequence information by UDPS down to HBV DNA levels of the order of 2-3 $\log _{10} \mathrm{IU} / \mathrm{mL}$. To avoid a bias linked to differences in the HBV DNA levels in different blood samples, we chose to express the results as absolute amounts of viral variants in each sample, in IU $/ \mathrm{mL}$, rather than proportions of the full quasispecies. In addition, the use of PyroDyn ${ }^{\odot}$ and PyroLink ${ }^{\odot}$ software allowed us to avoid a bias related to the HBV DNA amount in the sample, because only viral populations that were exponentially growing or decreasing over time in serial blood samples were detected and described, regardless of the HBV DNA content.

Pre-existence of resistant $\mathrm{HBV}$ variants in patients never exposed to nucleoside/nucleotide analogues is an accepted concept. However, classical techniques, such as population sequencing, reverse hybridization or even cloning of PCR products 
followed by sequencing of the clones, are generally not sensitive enough to detect variants present in very small proportions due to their poor relative fitness compared to wild-type virus. UDPS has been used to assess the pre-existence of HBV variants resistant to nucleoside/nucleotide analogues in a few studies. These works however suffered from important methodological flaws, including lack of sensitivity, no consideration of the error rate of the method to establish reliable cutoffs and ensure specificity, too short genomic region analyzed, and/or no linkage studies $(17,26,27)$.

Using UDPS, we found that variants with amino acid substitutions at positions rtA181 and rtN236 were already present as minor populations at baseline in most of the treatment-naïve patients who subsequently developed adefovir resistance, with a sensitivity $\leq 0.22 \%$. These substitutions were also detected during therapy in the remaining patients, suggesting that they may also have been present at baseline but in amounts too small to be detected by UDPS. The frequency of adefovir resistance substitutions at baseline may have been overestimated compared to the general population, as the patients we studied were selected because adefovir resistance occurred during treatment. In order to address this question, we tested at baseline two additional groups of patients, including a cohort of HBeAg-positive patients who seroconverted to anti-HBe and remained HBV DNA-undetectable after successful adefovir therapy, and a group of unselected treatment-naive patients newly seen in a tertiary referral center in France. In the latter group, which was comparable in age, gender and HBeAg status to our 7 patients who failed on adefovir therapy, a similar prevalence of rtA181V/T and rtN236T substitutions was found at baseline, ruling out an overestimation of the frequency of adefovir resistance substitutions in our study cohort. In the HBe seroconverter group, two patients harbored rtA181V/T substitutions, but none of them harbored the rtN236T substitution. This could suggest a lower frequency 
of UDPS-detectable amino acid substitutions in these young adults than in older patients at baseline, possibly due to the shorter duration of infection. Interpretation should however be extremely careful given the small number of patients studied in each group that did not allow for reliable statistical comparison. Substitutions at position rtM204, that confer cross-resistance to lamivudine, telbivudine and entecavir, were also found as minor populations at baseline in several patients from the 3 groups, whereas amino acid substitutions that confer resistance to entecavir when associated with rtM204 substitutions were more rarely found. These results were in keeping with the recent report, based on UDPS analysis, of preexisting amino acid substitutions known to confer resistance to nucleoside/nucleotide analogues in 3 patients who subsequently responded to this therapy and one patient who developed multidrug resistance (27). Larger-scale UDPS studies are now needed to assess the prevalence of primary resistance to nucleoside/nucleotide analogues in the general HBV-infected population.

During treatment, UDPS data analysis from a large number of serial samples obtained over several years allowed us to thoroughly characterize the complex dynamics of HBV populations on adefovir therapy, revealing successive waves of selection of HBV viral populations (Figures 1 and 2). Our findings can be summarized as follows.

(i) The dynamics of viral variants differed among the patients, despite the fact that they were receiving the same treatment, emphasizing the importance of the HBV quasispecies composition at the start of therapy and of individual viral variant fitness, both of which are patientspecific.

(ii) In patients 1 and 4, the wild-type virus rapidly declined at the beginning of treatment, whereas a few pre-existing variants did not 
appear to be affected, suggesting primary resistance. These variants were soon replaced by variants with single amino acid substitutions (including at position rtA181 or rtN236), which were in turn replaced by more complex variants with multiple amino acid substitutions.

(iii) Other patients exhibited a different pattern of resistance, with initial selection of variants with multiple substitutions, that were subsequently replaced by simpler variants.

(iv) Other substitutions, such as those at positions rt238 in patients 1, 4 and 6 (including reversion to $\mathrm{N}$ in patient 1) and at rtY245 in patient 1, appeared to be associated with a fitness gain of rtN236T variants. Interestingly, rtN238T has already been reported in two patients who developed resistance to adefovir $(12,28)$.

(v) Variants with substitutions at both rtA181 and rtN236 were present during follow-up in several patients and finally took over in two cases (patients 1 and 5).

(vi) The addition of lamivudine always reduced HBV DNA levels but did not alter the relative fitness of adefovir-resistant variants, which remained dominant during combination therapy. Interestingly, variants with rtA181V/T substitutions partially responded to lamivudine.

(vii) As expected, the rtA181V substitution was systematically associated with the sL173F substitution as a result of overlapping open reading frames coding for HBV reverse transcriptase and HBsAg. The rtA181T substitution was present in large amounts in patient 1 only and was associated with an sW172L substitution in 10\%-20\% of variants and a stop codon in the remaining $80 \%-90 \%$ of variants during therapy, 
suggesting transcomplementation of defective variants by sW172L variants. Positions sW172 and sL173 of HBsAg are located on the internal side of the membrane and are thus unlikely to play a role in the immune control of infection that may have influenced the fitness of the corresponding variants.

(viii) As shown in patient 1, substitutions in other genomic regions (such as HBsAg) not encoded by the nucleotide changes responsible for substitutions in the reverse transcriptase region selected by adefovir can be associated with the latter and play a role in viral fitness in vivo. This hypothesis is strengthened by the fact that the two selected substitutions, sS143T and sM197T, are located at the external side of HBsAg, within known immunodominant epitopes. However, this was seen in only one patient in this study.

Adefovir dipivoxil is still widely used worldwide, alone or in combination with lamivudine. As shown in this study, variants with amino acid substitutions known to confer resistance to various nucleoside/nucleotide analogues, including adefovir, can be detected in a substantial proportion of treatment-naïve patients with chronic hepatitis B. Larger-scale studies are now required to determine whether baseline testing with UDPS will be useful to orientate HBV treatment strategies. In addition, next-generation sequencing methods such as UDPS could be of considerable interest for early diagnosis of viral resistance during antiviral therapy. Indeed, with our approach, resistance could be diagnosed at approximately the same time as with cloning and sequencing (thus considerably earlier than with population sequencing), in a user-friendly and rapid way, compatible with clinical practice (data not shown). This will be facilitated in the future 
when the costs are reduced and the technology becomes easily available through specialized platforms.

In conclusion, using an original software package for analyzing viral sequences generated by UDPS and other next-generation sequencing methods in the context of antiviral resistance, (i) we showed that substitutions conferring HBV resistance to nucleoside/nucleotide analogues pre-exist in patients who have never been exposed to these drugs; (ii) we characterized the complex and heterogeneous dynamics of adefovirresistant viral populations in a group of $\mathrm{HBV}$-infected patients in whom resistance emerged during long-term adefovir therapy; (iii) we identified so far unknown amino acid substitutions that appeared to play an important role in HBV resistance to adefovir. These findings will also be helpful for understanding resistance to tenofovir, which shows cross-resistance with adefovir in vitro. Our findings imply that next-generation sequencing data analysis will have a number of applications in viral resistance assessment, as we recently reported with hepatitis $\mathrm{C}$ virus and human immunodeficiency virus $(29,30)$.

\section{ACKNOWLEDGEMENTS}

The authors thank Thierry Ravard for his help with mathematical modeling, Françoise Roudot-Thoraval for her help with statistical tests, Katyna Borroto-Esoda and Gilead Sciences for providing the samples from patients treated with adefovir used in this study, and Prof. Manh-Tong Dao for providing the samples from unselected patients newly seen in the Department of Hepatology of the University Hospital of Caen, France. 


\section{REFERENCES}

1. Ott JJ, Stevens GA, Groeger J, Wiersma ST. Global epidemiology of hepatitis B virus infection: new estimates of age-specific HBsAg seroprevalence and endemicity. Vaccine 2012;30:2212-2219.

2. Kim WR. Epidemiology of hepatitis $B$ in the United States. Hepatology 2009;49:S28-34.

3. McMahon BJ. The natural history of chronic hepatitis B virus infection. Hepatology 2009;49:S45-55.

4. Yang JD, Roberts LR. Hepatocellular carcinoma: A global view. Nat Rev Gastroenterol Hepatol 2010;7:448-458.

5. Zoulim F, Locarnini S. Hepatitis B virus resistance to nucleos(t)ide analogues. Gastroenterology 2009;137:1593-1608.

6. Pawlotsky JM. The concept of hepatitis B virus mutant escape. J Clin Virol 2005;34 (Suppl 1):S125-129.

7. Pawlotsky JM. Treatment failure and resistance with direct-acting antiviral drugs against hepatitis C virus. Hepatology 2011;53:1742-1751.

8. Lok AS, Zoulim F, Locarnini S, Mangia A, Niro G, Decraemer H, Maertens G, et al. Monitoring drug resistance in chronic hepatitis B virus (HBV)-infected patients during lamivudine therapy: evaluation of performance of INNO-LiPA HBV DR assay. J Clin Microbiol 2002;40:3729-3734.

9. Niesters HG, Zoulim F, Pichoud C, Buti M, Shapiro F, D'Heuvaert N, Celis L, et al. Validation of the INNO-LiPA HBV DR assay (version 2) in monitoring hepatitis B virus-infected patients receiving nucleoside analog treatment. Antimicrob Agents Chemother 2010;54:1283-1289. 
10. Pallier C, Castera L, Soulier A, Hezode C, Nordmann P, Dhumeaux D, Pawlotsky JM. Dynamics of hepatitis B virus resistance to lamivudine. J Virol 2006;80:643-653.

11. Pallier C, Rodriguez C, Brillet R, Nordmann P, Hezode C, Pawlotsky JM. Complex dynamics of hepatitis B virus resistance to adefovir. Hepatology 2009;49:50-59.

12. Yim HJ, Hussain M, Liu Y, Wong SN, Fung SK, Lok AS. Evolution of multi-drug resistant hepatitis B virus during sequential therapy. Hepatology 2006;44:703712.

13. Villet S, Pichoud C, Villeneuve JP, Trepo C, Zoulim F. Selection of a multiple drugresistant hepatitis B virus strain in a liver-transplanted patient. Gastroenterology 2006;131:1253-1261.

14. Margulies M, Egholm M, Altman WE, Attiya S, Bader JS, Bemben LA, Berka J, et al. Genome sequencing in microfabricated high-density picolitre reactors. Nature $2005 ; 437: 376-380$.

15. Petrosino JF, Highlander S, Luna RA, Gibbs RA, Versalovic J. Metagenomic pyrosequencing and microbial identification. Clin Chem 2009;55:856-866.

16. Margeridon-Thermet S, Shulman NS, Ahmed A, Shahriar R, Liu T, Wang C, Holmes SP, et al. Ultra-deep pyrosequencing of hepatitis B virus quasispecies from nucleoside and nucleotide reverse-transcriptase inhibitor (NRTI)-treated patients and NRTI-naive patients. J Infect Dis 2009;199:1275-1285.

17. Solmone M, Vincenti D, Prosperi MC, Bruselles A, Ippolito G, Capobianchi MR. Use of massively parallel ultradeep pyrosequencing to characterize the genetic diversity of hepatitis $B$ virus in drug-resistant and drug-naive patients and to detect minor variants in reverse transcriptase and hepatitis B S antigen. J Virol 2009;83:1718-1726. 
18. Hadziyannis SJ, Tassopoulos NC, Heathcote EJ, Chang TT, Kitis G, Rizzetto M, Marcellin P, et al. Long-term therapy with adefovir dipivoxil for HBeAg-negative chronic hepatitis B for up to 5 years. Gastroenterology 2006;131:1743-1751.

19. Jonas MM, Kelly D, Pollack H, Mizerski J, Sorbel J, Frederick D, Mondou E, et al. Safety, efficacy, and pharmacokinetics of adefovir dipivoxil in children and adolescents (age 2 to $<18$ years) with chronic hepatitis B. Hepatology 2008;47:1863-1871.

20. Pichoud C, Seigneres B, Wang Z, Trepo C, Zoulim F. Transient selection of a hepatitis B virus polymerase gene mutant associated with a decreased replication capacity and famciclovir resistance. Hepatology 1999;29:230-237.

21. Altshuler D, Pollara VJ, Cowles CR, Van Etten WJ, Baldwin J, Linton L, Lander ES. An SNP map of the human genome generated by reduced representation shotgun sequencing. Nature 2000;407:513-516.

22. Smith TF, Waterman MS. Identification of common molecular subsequences. J Mol Biol 1981;147:195-197.

23. Campbell PJ, Pleasance ED, Stephens PJ, Dicks E, Rance R, Goodhead I, Follows GA, et al. Subclonal phylogenetic structures in cancer revealed by ultra-deep sequencing. Proc Natl Acad Sci USA 2008;105:13081-13086.

24. Metzker ML. Sequencing technologies-the next generation. Nat Rev Genet 2010;11:31-46.

25. Chevaliez S, Rodriguez C, Pawlotsky JM. New virologic tools for management of chronic hepatitis B and C. Gastroenterology 2012;142:1303-1313.

26. Mello FC, Lago BV, Lewis-Ximenez LL, Fernandes CA, Gomes SA. Detection of mixed populations of wild-type and YMDD hepatitis $B$ variants by 
pyrosequencing in acutely and chronically infected patients. BMC Microbiol $2012 ; 12: 96$.

27. Rodriguez-Frias F, Tabernero D, Quer J, Esteban JI, Ortega I, Domingo E, Cubero $M$, et al. Ultra-deep pyrosequencing detects conserved genomic sites and quantifies linkage of drug-resistant amino acid changes in the hepatitis B virus genome. PLoS One 2012;7:e37874.

28. Villet S, Pichoud C, Billioud G, Barraud L, Durantel S, Trepo C, Zoulim F. Impact of hepatitis B virus rtA181V/T mutants on hepatitis B treatment failure. J Hepatol 2008;48:747-755.

29. Rodriguez C, Soulie C, Marcelin AG, Calvez V, Descamps D, Charpentier C, Flandre P, et al. HIV-1 coreceptor usage prediction by ultra-deep pyrosequencing increases the rate of patients treated with success by CCR 5 antagonists. Antiviral Ther 2011;16 (Suppl 1):A100.

30. Chevaliez S, Rodriguez C, Soulier A, Ahmed-Belkacem A, Hezode C, Pawlotsky JM. Molecular characterization of HCV resistance to telaprevir by means of ultra-deep pyrosequencing: preexisting resistant variants and dynamics of resistant populations. J Hepatol 2011;54 (Suppl 1):S30. 


\section{FIGURE LEGENDS}

Fig. 1. Dynamics of viral variants on adefovir-based antiviral therapy in patient 1. (A) Dynamics of HBV viral variants as assessed by analysis of UDPS data on the reverse transcriptase domain. (B) Combined analysis of UDPS data on both the reverse transcriptase and HBsAg domains, including amino acid substitutions at positions $172 / 173$ of the s region associated with $\mathrm{rtA} 181 \mathrm{~V} / \mathrm{T}$ substitutions. sW172* indicates conversion of a tryptophan codon into a stop codon at position 172 . Results are expressed as the absolute amount of each $\mathrm{HBV}$ variant in $\log _{10} \mathrm{IU} / \mathrm{mL}$ at each time point. Dynamics of HBV DNA levels are shown at the back in blue.

\section{Fig. 2. Dynamics of viral variants on adefovir-based antiviral therapy in patients 2}

to 7. Results are expressed as the absolute amount of each HBV variant in $\log _{10} \mathrm{IU} / \mathrm{mL}$ at each time point in patient $2(A)$, patient $3(B)$, patient $4(C)$, patient $5(D)$, patient $6(E)$ and patient 7 (F). Dynamics of HBV DNA levels are shown at the back in blue. 
Table 1. Prevalence of amino acid substitutions known to confer HBV resistance to nucleoside/nucleotide analogues at baseline, as detected by UDPS in the 7 patients who failed on adefovir therapy from this study, in a comparator cohort of patients who succeeded on adefovir therapy (HBe seroconversion and sustained undetectable HBV DNA) and in a comparator cohort of unselected, untreated treatment-naïve subjects with chronic hepatitis B. <: frequency of the substitution was below the calculated detection cutoff which is indicated at the bottom of the table for each position. rtM204V/I confers cross-resistance to lamivudine, telbivudine, and entecavir; rtV173L and rtL180M confer improved fitness to rtM204V/I variants; rtN236T confers resistance to adefovir; rtA181V/T confers crossresistance to lamivudine, telbivudine and adefovir; rtT184S/A/I/L and rtR202G confer additional resistance to entecavir and improved fitness to variants bearing rtM204V/I, in the presence of this drug.

\begin{tabular}{|c|c|c|c|c|c|c|c|c|c|}
\hline Cohorts & Patients & $\begin{array}{c}\text { HBV DNA } \\
\text { level (Log } \\
\text { IU/mL) }\end{array}$ & rtV173L & rtL180M & $\mathrm{rtA181V/T}$ & $\begin{array}{c}\mathrm{rtT} 184 \\
\mathrm{~S} / \mathrm{A} / \mathrm{I} / \mathrm{L}\end{array}$ & rtR202G & $\begin{array}{c}\text { rtM204 } \\
\text { V/I }\end{array}$ & rtN236T \\
\hline \multirow{6}{*}{ 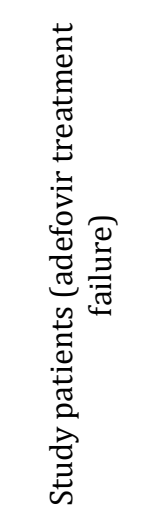 } & Patient 1 & 6.6 & $<$ & $<$ & $0.47 \%$ & $<$ & $<$ & $0.31 \%$ & $0.25 \%$ \\
\hline & Patient 2 & 7.8 & $<$ & $<$ & $0.18 \%$ & $<$ & $<$ & $<$ & $<$ \\
\hline & Patient 3 & 6.8 & $<$ & $<$ & $0.22 \%$ & $<$ & $<$ & $0.32 \%$ & $<$ \\
\hline & Patient 5 & 7.6 & $<$ & $<$ & $0.33 \%$ & $<$ & $<$ & $<$ & $<$ \\
\hline & Patient $6^{*}$ & 6.2 & $<$ & $<$ & $<$ & $<$ & $<$ & $<$ & $<$ \\
\hline & Patient 7 & 6.1 & $<$ & $<$ & $0.33 \%$ & $<$ & $<$ & $<$ & $<$ \\
\hline
\end{tabular}




\begin{tabular}{|c|c|c|c|c|c|c|c|c|c|}
\hline \multirow{4}{*}{ 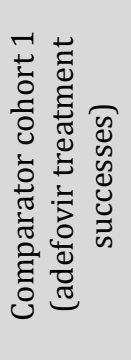 } & Patient A & 5.2 & $<$ & $<$ & $0.47 \%$ & $<$ & $<$ & $<$ & $<$ \\
\hline & Patient C & 7.1 & $<$ & $6.32 \%$ & $<$ & $<$ & $<$ & $9.57 \%$ & $<$ \\
\hline & Patient D & 6.4 & $<$ & $<$ & $<$ & $<$ & $0.31 \%$ & $<$ & $<$ \\
\hline & Patient E & 5.7 & $<$ & $<$ & $0.39 \%$ & $<$ & $<$ & $0.37 \%$ & $<$ \\
\hline \multirow{8}{*}{ 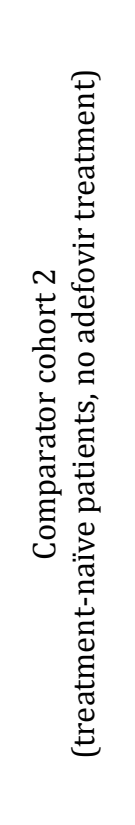 } & Patient $\mathrm{AB}$ & 3.6 & $<$ & $<$ & $<$ & $0.35 \%$ & $<$ & $0.32 \%$ & $<$ \\
\hline & Patient AC & 3.7 & $<$ & $<$ & $<$ & $0.29 \%$ & $<$ & $0.34 \%$ & $1.88 \%$ \\
\hline & Patient AD & 2.8 & $<$ & $<$ & $<$ & $<$ & $<$ & $0.27 \%$ & $<$ \\
\hline & Patient AE & 3.7 & $<$ & $<$ & $<$ & $<$ & $<$ & $<$ & $<$ \\
\hline & Patient AH & 2.1 & $<$ & $<$ & $<$ & $0.34 \%$ & $<$ & $1.79 \%$ & $<$ \\
\hline & Patient AI & 3.2 & $<$ & $<$ & $<$ & $<$ & $<$ & $<$ & $0.60 \%$ \\
\hline & Patient AJ & 7.1 & $<$ & $<$ & $0.30 \%$ & $0.30 \%$ & $<$ & $1.02 \%$ & $0.64 \%$ \\
\hline & Patient AK & 4.1 & $<$ & $<$ & $<$ & $<$ & $<$ & $<$ & $<$ \\
\hline- & $\begin{array}{c}\text { Detection } \\
\text { cutoff of the } \\
\text { substitution }\end{array}$ & - & $0.05 \%$ & $0.03 \%$ & $0.08 \%$ & $0.15 \%$ & $0.18 \%$ & $0.21 \%$ & $0.22 \%$ \\
\hline
\end{tabular}

*The number of sequences obtained with the baseline sample was smaller in this patient than in the others, possibly impacting sensitivity of detection.

『The detection cutoff of each substitution was calculated as the mean maximal error rate for the given substitution plus two standard deviations. 


\section{Figure 1A}

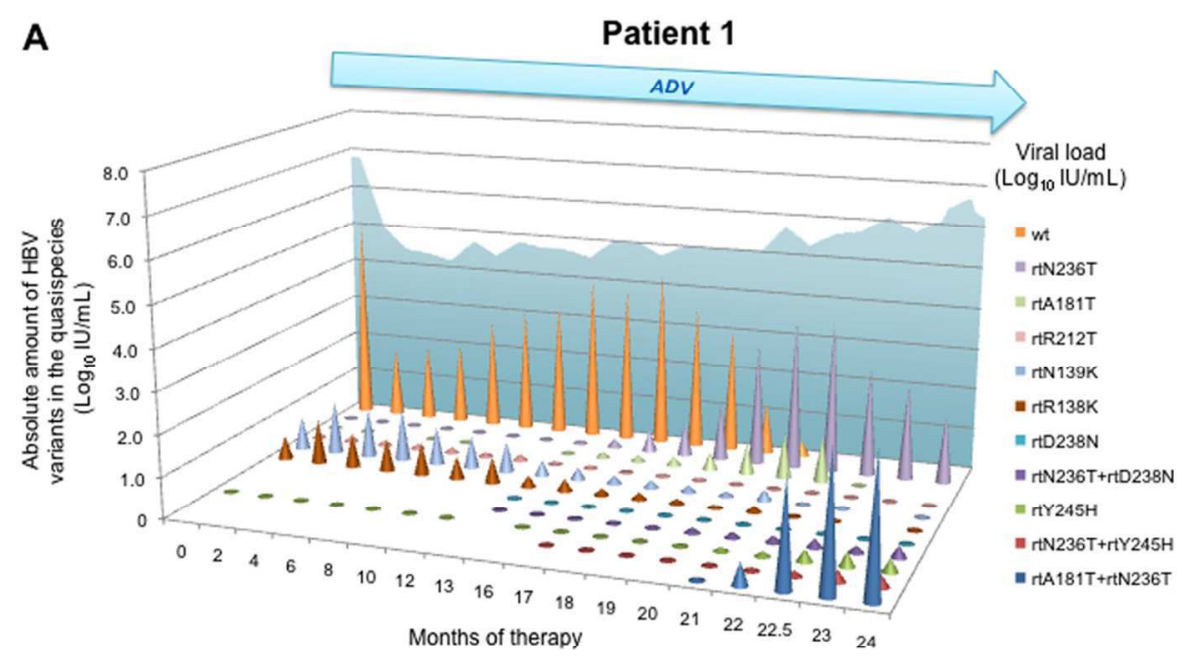

Figure $1 \mathrm{~A}$

$99 \times 75 \mathrm{~mm}(300 \times 300 \mathrm{DPI})$ 
Figure $1 \mathrm{~B}$

$99 \times 75 \mathrm{~mm}(300 \times 300$ DPI $)$

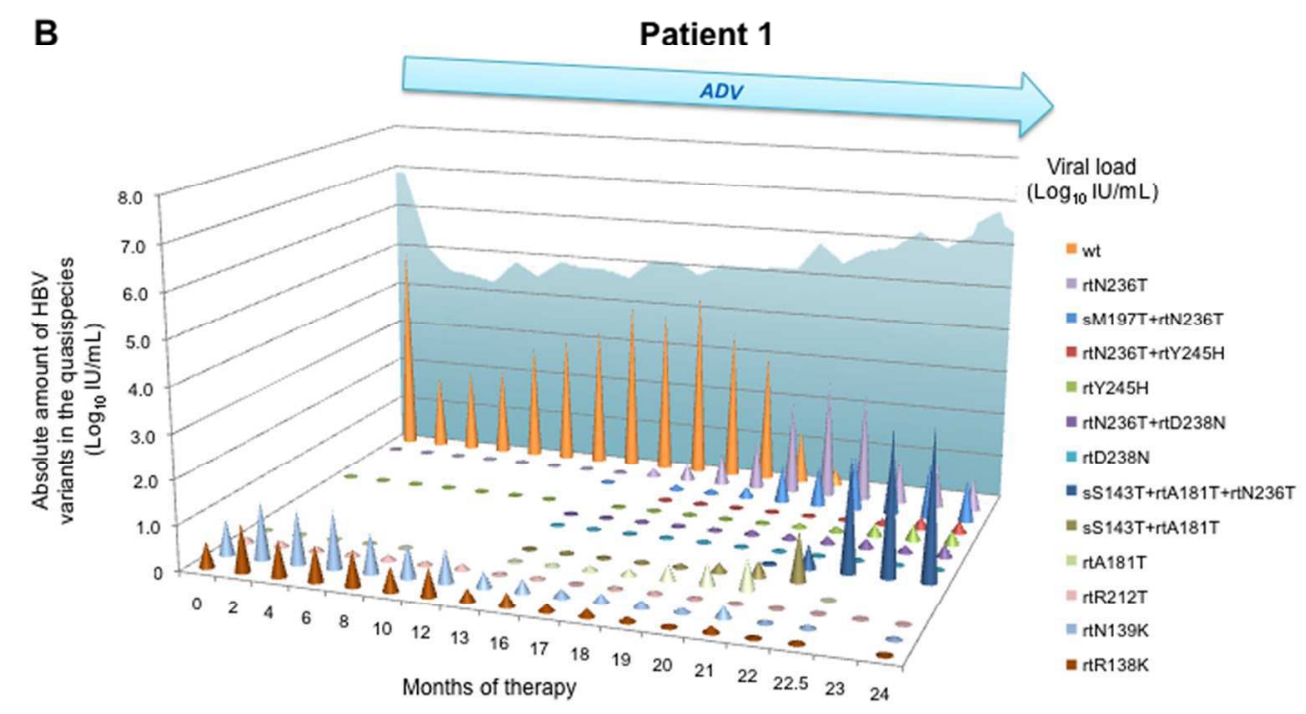

\section{Figure 1B}


Figure 2A

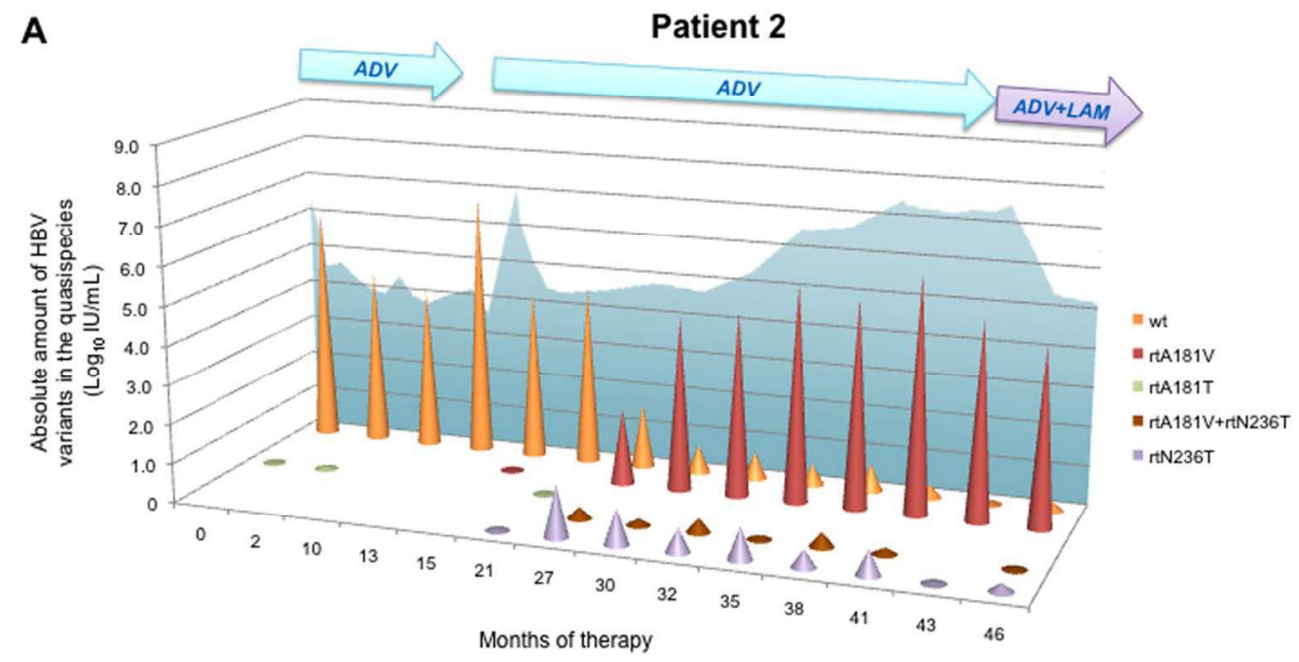

Figure $2 \mathrm{~A}$

$99 \times 75 \mathrm{~mm}(300 \times 300 \mathrm{DPI})$ 
Figure 2B

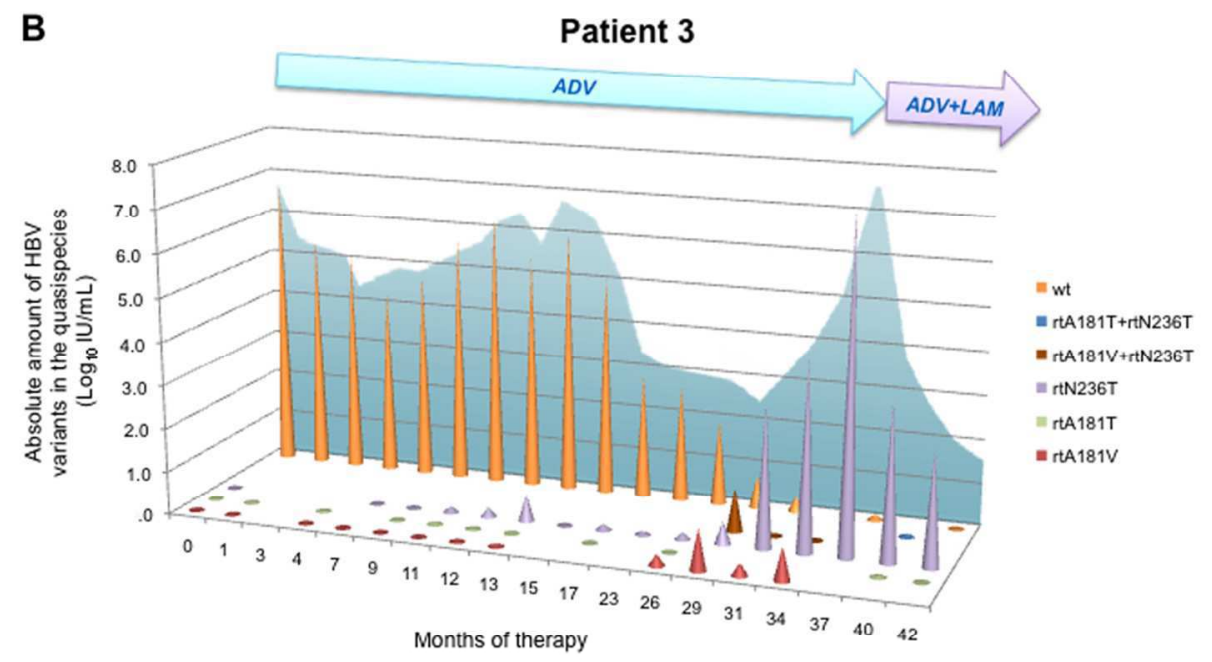

Figure 2B

$99 \times 75 \mathrm{~mm}(300 \times 300 \mathrm{DPI})$ 
Figure 2C

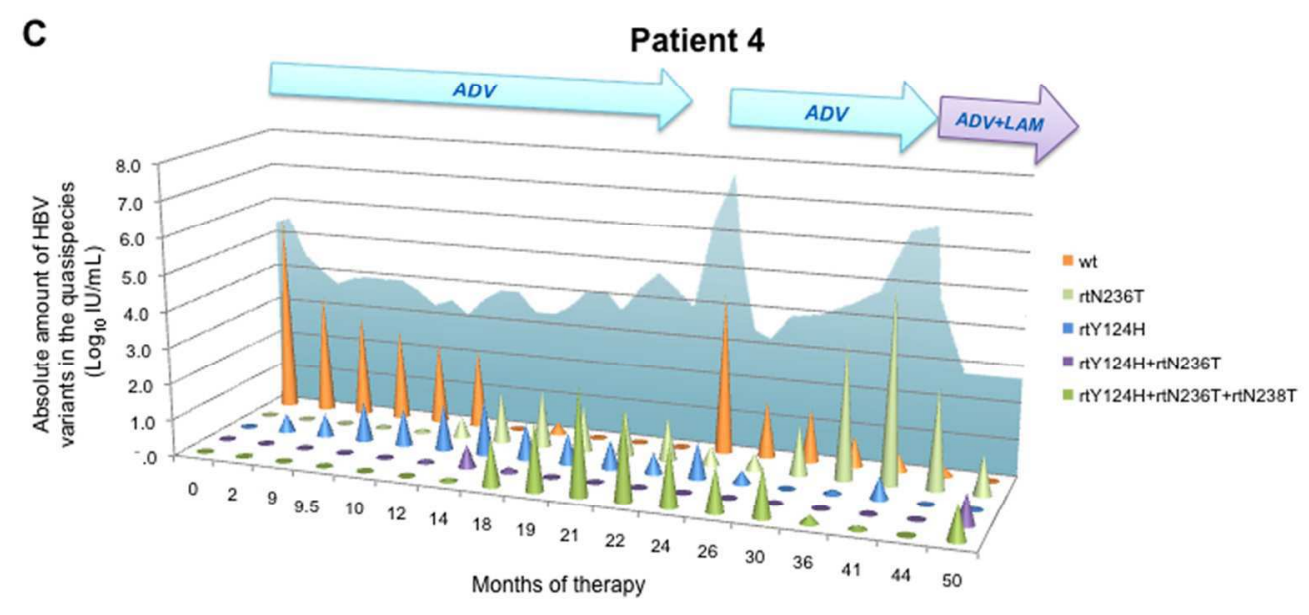

Figure $2 \mathrm{C}$

$99 \times 75 \mathrm{~mm}(300 \times 300$ DPI $)$ 
1

2

3

4

5

6

7

8

9

10

11

12

13

14

15

16

17

18

19

20

21

22

23

24

25

26

27

28

29

30

31

32

33

34

35

36

37

38

39

40

41

42

43

44

45

46

47

48

49

50

51

52

53

54

55

56

57

58

59

60

Figure 2D

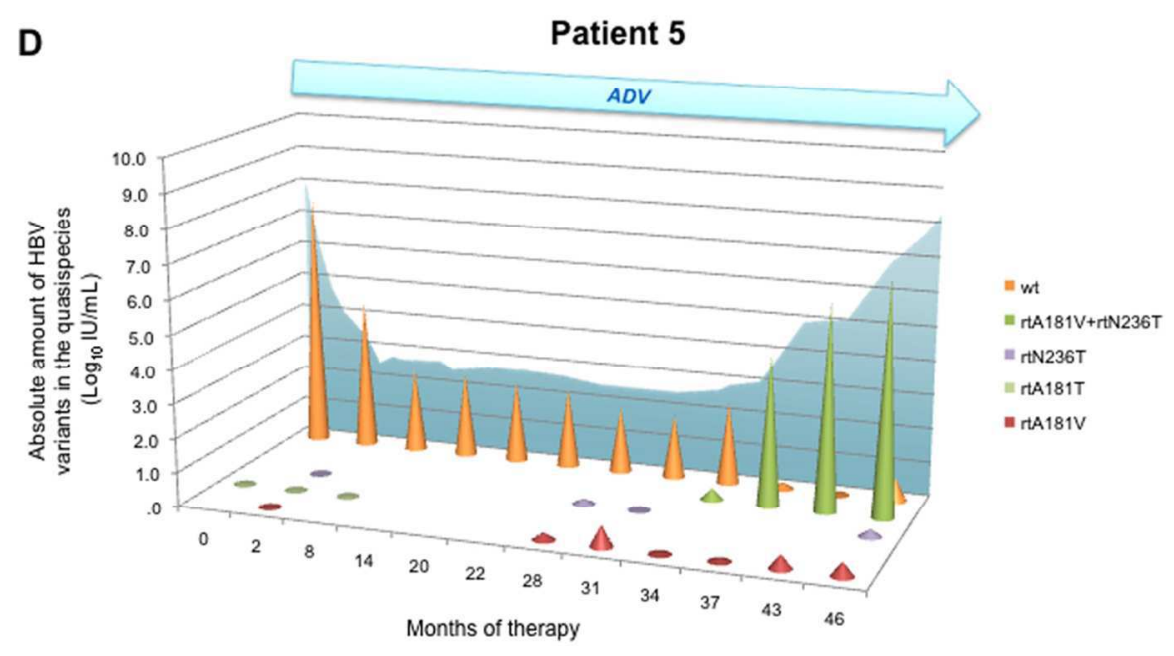

Figure 2D

$99 \times 75 \mathrm{~mm}(300 \times 300 \mathrm{DPI})$ 


\section{Figure 2E}

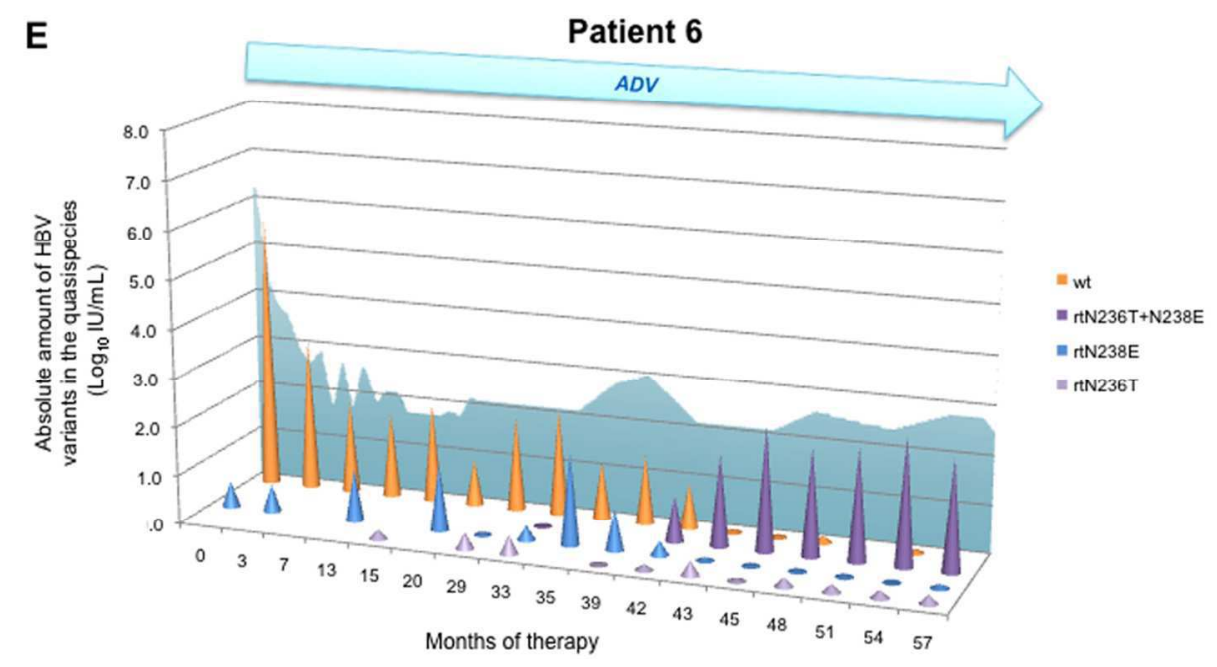

Figure 2E

$99 \times 75 \mathrm{~mm}(300 \times 300$ DPI $)$ 
Figure 2F

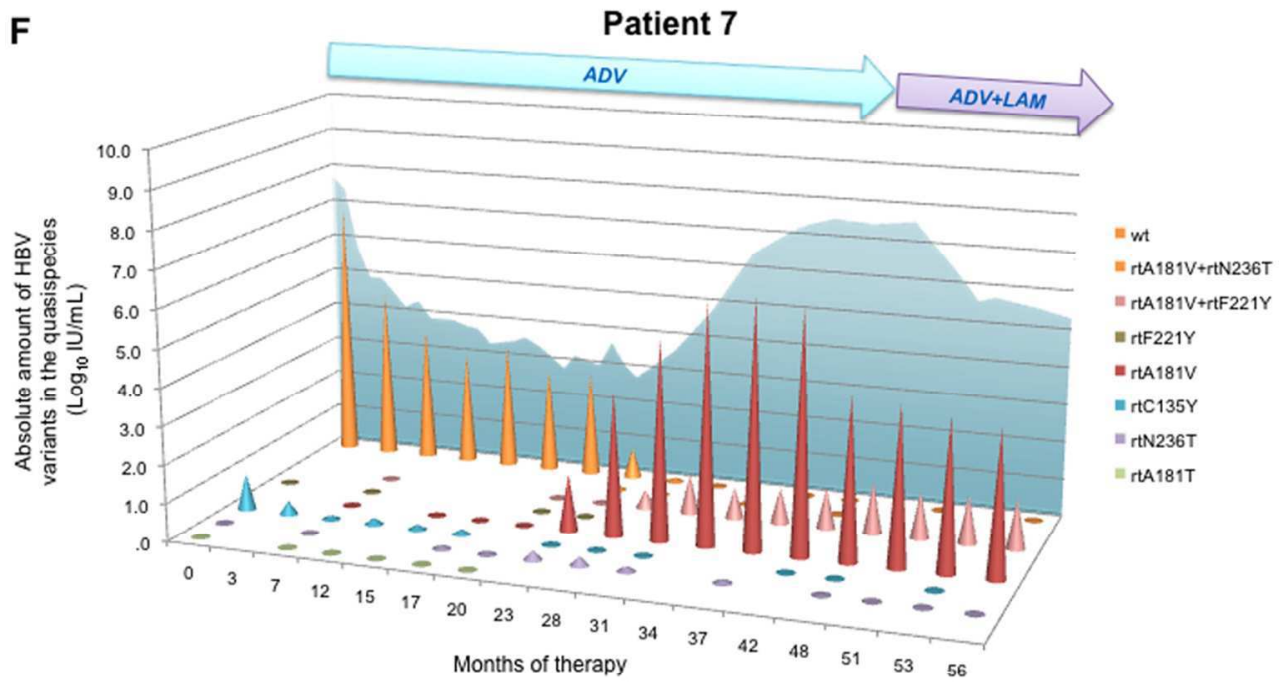

Figure $2 \mathrm{~F}$

$99 \times 75 \mathrm{~mm}(300 \times 300$ DPI $)$ 
Supplementary Table 1. Numbering of the patients in Pallier et al., Hepatology 2009;49:50-59 and in the present article

\begin{tabular}{|l|l|}
\hline Rodriguez et al., 2013 & Pallier et al., 2009 \\
\hline Patient 1 & Patient D \\
\hline Patient 2 & Patient E \\
\hline Patient 3 & Patient B \\
\hline Patient 4 & Patient G \\
\hline Patient 5 & Patient C \\
\hline Patient 6 & Patient F \\
\hline Patient 7 & Patient A \\
\hline
\end{tabular}

